\title{
Principais fatores de risco para delirium encontrados nos pacientes idosos internados nas enfermarias de clínica médica de um hospital da Amazônia
}

Main risk factors for delirium found in elderly patients inside the nurses of medical clinics of an amazon hospital

Principales factores de riesgo para delirium encontrados en los pacientes idosos internados en las enfermarias de clínica médica de un hospital de la amazonia

Rudival Faial de Moraes Junior ${ }^{1}$, Andrea Negrão Costa ${ }^{1}$, Rodrigo Bona Maneschy ${ }^{1}$, Carla Daniele Nascimento Pontes ${ }^{1 *}$, Yuri José Almeida da Silva ${ }^{1}$, Lucianna Serfaty de Holanda ${ }^{1}$, Leonardo Santos da Silva ${ }^{1}$, Luis Gustavo Silva Lima ${ }^{1}$, Amanda Freitas Teixeira da Silva², Aline Dutra Valente Duarte ${ }^{2}$, Danilo Rabelo Mendes², Vitor Bruno Teixeira de Holanda'1.

\section{RESUMO}

Objetivo: Identificar os principais fatores de risco para delírium encontrados nos pacientes idosos internados nas enfermarias de clínica médica em um Hospital da Amazônia. Metodologia: Trata-se de um estudo descritivo, transversal, que realizou entrevista e consulta em prontuário dos pacientes que apresentaram delírium e que aceitaram participar da pesquisa. Resultados: A maioria dos pacientes era do sexo masculino, todos os pacientes possuíam fatores de risco predisponentes e precipitantes, todos possuíam acima de 65 anos. Os principais fatores de risco predisponentes foram Idade avançada, Polifarmácia e quedas e os fatores precipitantes mais prevalentes foram uso de inibidor de bomba de protóns, infecção e dor. Conclusão: É necessário aperfeiçoar o exame clínico da equipe de saúde que lida diretamente com os pacientes idosos, a fim de detectar precocemente os sinais que sugerem uma evolução para o estado de delirium e estabelecer medidas terapêuticas e preventivas, para melhorar $o$ atendimento aos pacientes e evitar as complicações.

Palavras-Chave: Delirium, Fatores Precipitantes, Fatores Predisponentes.

\begin{abstract}
Objective: To identify the main risk factors for delirium found in elderly patients hospitalized in the medical clinic at a Hospital da Amazônia.Methodology: This is a descriptive, cross-sectional study that interviewed and consulted in the medical records of patients who presented delirium and who accepted to participate in the study. Results: Most of the patients were male, all patients had predisposing and precipitating risk factors, all of them over 65 years old. The main predisposing risk factors were advanced age, polypharmacy and falls, and the most prevalent precipitating factors were use of proton pump inhibitor, infection and pain. Conclusion: It is necessary to improve the clinical examination of the health team that deals directly with the
\end{abstract}

${ }^{1}$ Fundação Santa Casa de Misericórdia do Pará. * E-mail: pontes4@live.com

2Universidade do Estado do Pará.

SUBMETIDO EM: 10/2018 | ACEITO EM: 11/2018

I PUBLICADO EM: 01/2019

REAS/EJCH | Vol.Sup.17 | e272 | DOI: https://doi.org/10.25248/reas.e272.2019 Página 1 de 8 
elderly patients, in order to detect early signs of delirium progression and establish therapeutic and preventive measures to improve patient care and avoid complications.

Keywords: Delirium, Precipitating Factors, Predisposing Factors.

\section{RESUMEN}

Objetivo: Identificar los principales factores de riesgo para delirio encontrados en los pacientes ancianos internados en las enfermerías de clínica médica en un Hospital de la Amazonia. Metodología: Se trata de un estudio descriptivo, transversal, que realizó entrevista y consulta en prontuario de los pacientes que presentaron delirio y que aceptaron participar de la investigación. Resultados: La mayoría de los pacientes eran del sexo masculino, todos los pacientes tenían factores de riesgo predisponentes y precipitantes, todos tenían más de 65 años. Los principales factores de riesgo predisponentes fueron Edad avanzada, Polifarmacia y caídas y los factores precipitantes más prevalentes fueron uso de inhibidor de bomba de protóns, infección y dolor. Conclusíon: Es necesario perfeccionar el examen clínico del equipo de salud que se dirige directamente con los pacientes ancianos, a fin de detectar precozmente las señales que sugieren una evolución hacia el estado de delirium y establecer medidas terapéuticas y preventivas, para mejorar la atención a los pacientes y evitar las complicaciones.

Palabras clave: Delirium, Factores precipitantes, Factores preponderantes.

\section{INTRODUÇÃO}

O delirium é a alteração cognitiva confusional aguda mais prevalente em idosos. Em idosos na comunidade ocorre em $1 \%$ a $14 \%$, entre os pacientes hospitalizados $14 \%$ a $56 \%$, chegando até $70 \%$ a $87 \%$ em unidade de terapia intensiva. (GORZONI e FABBRI, 2013). O delirium foi uma das primeiras doenças mentais descritas na literatura médica. Nos trabalhos de Hipócrates (460-366 a.c), encontram-se inúmeras referências ao conjunto de sintomas que hoje chamamos de delirium. Portanto, apesar de ser uma entidade nosológica caracterizada há mais de 2.500 anos, continua sendo mal compreendida do ponto de vista fisiopatológico. (BRUNNER e SUDDARTH, 2011).

Define-se como uma síndrome orgânica cerebral caracterizada pela flutuação do estado mental, inatenção, alteração do nível de consciência (ou pensamento desorganizado), sem etiologia específica que acometem o paciente de maneira súbita, a qual pode ser classificada em três estágios: hiperativo, hipoativo e misto. Além disso, é uma patologia multifatorial, dentre os quais podem ser categorizados de três maneiras: características da própria doença aguda, fatores relacionados com o paciente e fatores ambientais e/ou iatrogênicos (TOMMASO et al 2016).

O Confusion Assessment Method (CAM) é um instrumento desenvolvido para rastrear delirium, sendo de simples e rápida aplicação, podendo ser aplicado no ambulatório ou na beira do leito, não se prestando a avaliar gravidade do delirium. Busca-se a presença de quatro critérios clínicos: A) Estado confusional agudo com flutuação marcante, B) Déficit de atenção marcante, C) Pensamento e discurso desorganizados, D) Alteração do nível de consciência (hipoativo ou hiperativo). Considera-se delirium na presença dos itens "A" e "B" mais "C" e/ou "D" (LÔBO et al., 2010).

No que diz respeito aos fatores precipitantes do delirium, os principais são as medicações (40\%) com potencial psicoativo conhecido, como as sedativo-hipnóticas (risco de delirium aumentado em 3 a 12 vezes), os narcóticos (em 3 vezes) e os anticolinérgicos (em 5 a 12 vezes); bem como fatores ambientais e psicossociais; infecções

REAS/EJCH | Vol.Sup.17 | e272 | DOI: https://doi.org/10.25248/reas.e272.2019 Página 2 de 8 
ocultas; falência respiratória; distúrbios endócrinos; e eventos iatrogênicos que correspondem em $29 \%$ a $38 \%$ dos idosos hospitalizados, são ocorrências relacionadas às complicações de diagnóstico e tratamento, como reações alérgicas e sangramentos (FREITAS e PY, 2016).

Delirium em alguns casos pode ser desencadeado por fator isolado, mas é mais comum a inter-relação entre condições que frequentemente provocam e fatores que agravam ou aumentam o risco de delirium. Além disso, os efeitos dos diversos fatores de risco parecem ser cumulativos. Assim, dentre os fatores que provocam delirium tem-se: infecções (pneumonia, erisipela, exarcebação de DPOC), patologias cardiovasculares (infarto, fibrilação atrial aguda, angina), cerebrovasculares (ataque isquêmico transitório, acidente vascular cerebral isquêmico), musculoesqueléticas (fraturas, luxações, entorses), endócrinas (descompensação diabética, hipo e hipertireoidismo), distúrbios hidroeletrolíticos (desidratação, hiponatremia, hipercalemia), medicamentos (anticolinérgicos, sedativos, narcóticos). Dentre os fatores agravantes tem-se: privação do sono, desidratação e déficit auditivo. (CHAIMOWICZ et al, 2013).

O diagnóstico diferencial é feito principalmente com demência, depressão e psicoses funcionais (GORZONI e FABBRI, 2013). A demência é uma síndrome que se caracteriza pelo declínio da memória associado a déficit de, pelo menos, uma outra função cognitiva (linguagem, gnosias, praxias ou funções executivas) com intensidade suficiente para interferir no desempenho social ou profissional do indivíduo. (SCHLINDWEIN-ZANINI,2010).

Já a depressão em pacientes idosos, além dos sintomas comuns, costuma ser acompanhada por queixas somáticas, hipocondria, baixa auto-estima, sentimentos de inutilidade, humor disfórico, tendência autodepreciativa, alteração do sono e do apetite, ideação paranóide e pensamento recorrente de suicídio. (STELLA et al, 2002). As psicoses funcionais têm início em faixas etárias mais precoces, com início súbito, porém sem flutuação de sintomas, alucinações predominantemente auditivas e ideias delirantes sustentadas (GORZONI e FABBRI, 2013).

Diante a frequência de pacientes com delirium terem maior risco de complicações, como pneumonia, úlcera de decúbito e complicações pós-cirúrgicas, assim como internações mais longas e pior prognóstico. (SOUSAMUÑOZ et al., 2012). Surgiu a necessidade de se avaliar a incidência e a prevalência em pacientes idosos asilados nas enfermarias de clínica médica da Fundação Santa Casa de Misericórdia do Pará (FSCMP), procurando detectar os fatores mais prevalentes como causa dessa comorbidade.

\section{METODOLOGIA}

De acordo com a resolução 466/12 do Conselho Nacional de Saúde e com Comitê de Ética em Pesquisa da Fundação Santa Casa de Misericórdia do Pará (FSCMP), foi solicitada analise do projeto de pesquisa e somente após aprovação do CEP, conforme parecer de número 2.147.360 é que foram iniciados os procedimentos para a coleta de dados.

A presente pesquisa é um estudo observacional descritivo transversal, com abordagem na identificação do perfil epidemiológico dos pacientes idosos hospitalizados na FSCMP que preencham critérios de Delirium, bem como estabelecer uma análise quantitativa e qualitativamente dos fatores de risco mais prevalentes no desencadeamento e manutenção dessa condição clínica.

Foram entrevistados os pacientes nas dependências das Enfermarias de Clínica Médica São Paulo e São Roque da Fundação Santa Casa de Misericórdia do Pará que tiverem apresentado pelo menos um episódio de Delirium durante o período de Junho de 2017 a Junho de 2018 e que concordaram que alguns dos seus dados relacionados a fatores de risco predisponentes para a condição de Delirium fossem retirados de seus prontuários.

Inicialmente, foram analisados os critérios de inclusão e exclusão dos pacientes internados. Em seguida, foi solicitado o aceite em participar da pesquisa por meio da assinatura do o Termo de Consentimento Livre e

REAS/EJCH | Vol.Sup.17 | e272 | DOI: https://doi.org/10.25248/reas.e272.2019 Página 3 de 8 
Esclarecido (TCLE), seja pelo paciente e/ou pelo seu responsável e após essa etapa será aplicado o questionário para investigação dos fatores de risco predisponentes e precipitantes para desenvolvimento de Delirium que o paciente apresentava durante o período de sua internação.

Os dados foram coletados por meio de instrumentos de pesquisa aplicados pelos próprios pesquisadores aos indivíduos participantes da pesquisa ou aos seus responsáveis, de forma a facilitar a obtenção das informações necessárias, especialmente em indivíduos com baixa escolaridade ou com algum comprometimento cognitivo.

Além disso, alguns dados foram coletados diretamente no prontuário dos pacientes, sobretudo em situações em que estes não possam ou não saibam responder no momento da entrevista, seguindo a relação dos fatores precipitantes e predisponentes levantados na entrevista.

Foram utilizados testes estatísticos como QiQuadrado e T-Student para efeito de cálculo e cruzamento de dados e para análise de relevância do trabalho buscando um $p$ valor $<0,05 \%$.

\section{RESULTADOS}

Foram identificados 13 pacientes com quadro de Delirium, dos 45 pacientes internados na clínica médica da FSCMPA, segundo os critérios CAM I - DSM 5 (Confusion Assessment Method - Manual Diagnóstico em Saúde Mental), o que releva uma taxa de incidência de $28 \%$. Desses pacientes $40 \%$ eram mulheres e $60 \%$ eram homens. A maioria tinha idade superior aos 65 anos, 100\% possuíam fatores precipitantes e 100\% possuíam fatores predisponentes para desenvolverem Delírium.

Os fatores precipitantes avaliados foram: Idade > 65 anos, sexo masculino, demência, déficit cognitivo, depressão, desidratação, doença hepática, imobilidade, abuso de álcool, histórico de delírium, traumas ou fraturas, dependência funcional, HIV, déficits sensoriais, queda, incontinência, doença renal, AVE e doenças neurológicas.

Os fatores predisponentes foram: Uso de medicamentos sedativos, anticolinérgicos, uso de fármacos para incontinência, uso de inibidores de bomba de prótons, infecções, choque, hipoxemia, infarto agudo do miocárdio, insuficiência renal e hepática, febre, retenção urinária ou intestinal, insuficiência cardíaca, desnutrição, distúrbios metabólicos e doenças endócrinas.

Tabela 1 - Perfil dos pacientes com quadro de delirium na enfermaria de clínica médica da FSCMPA entre julho-2017 e julho-2018.

\begin{tabular}{lcc}
\hline \multirow{2}{*}{ Características } & \multicolumn{2}{c}{ Pacientes (total = 13) } \\
\cline { 2 - 3 } & $\mathbf{n}$ & $\%$ \\
\hline Sexo & 5 & 38,46 \\
Feminino & 8 & 61,53 \\
Masculino & 13 & 100 \\
Idade $\geq 60$ anos & & \\
Fatores de risco & 13 & 100 \\
Predisponentes & 13 & 100 \\
Precipitantes & \\
\hline
\end{tabular}

Fonte: Protocolo de pesquisa. 
As informações foram coletas com os acompanhantes em $100 \%$ dos casos e os tipos de Delírium foram principalmente hipoativos, seguido do hiperativo e por fim o misto (Gráfico 1).

Gráfico 1 - Distribuição dos pacientes conforme o tipo de delirium apresentado na enfermaria de clínica médica da FSCMPA entre julho-2017 e julho-2018.



- Delirium Hipoativo

- Delirium Hiperativo

- Delírium Misto

Fonte: Protocolo de pesquisa

Dentre os Fatores Predisponentes, o mais prevalente foi idade superior a 65 anos, com prevalência de $90 \%$ dentre os pacientes, seguido de Polifarmácia com $80 \%$ e de quedas com $70 \%$ (Gráfico 2). Notou-se também um considerável número de idosos com Déficit Cognitivo (60\% dos pacientes), Imobilidade (60\% dos pacientes) e pacientes com múltiplas comorbidades (50\% dos pacientes) (Gráfico 2).

Gráfico 2 - Distribuição dos Fatores Predisponentes apresentados pelos pacientes com Delírium na Clínica médica da FSCMPA entre junho-2017 a junho-2018.

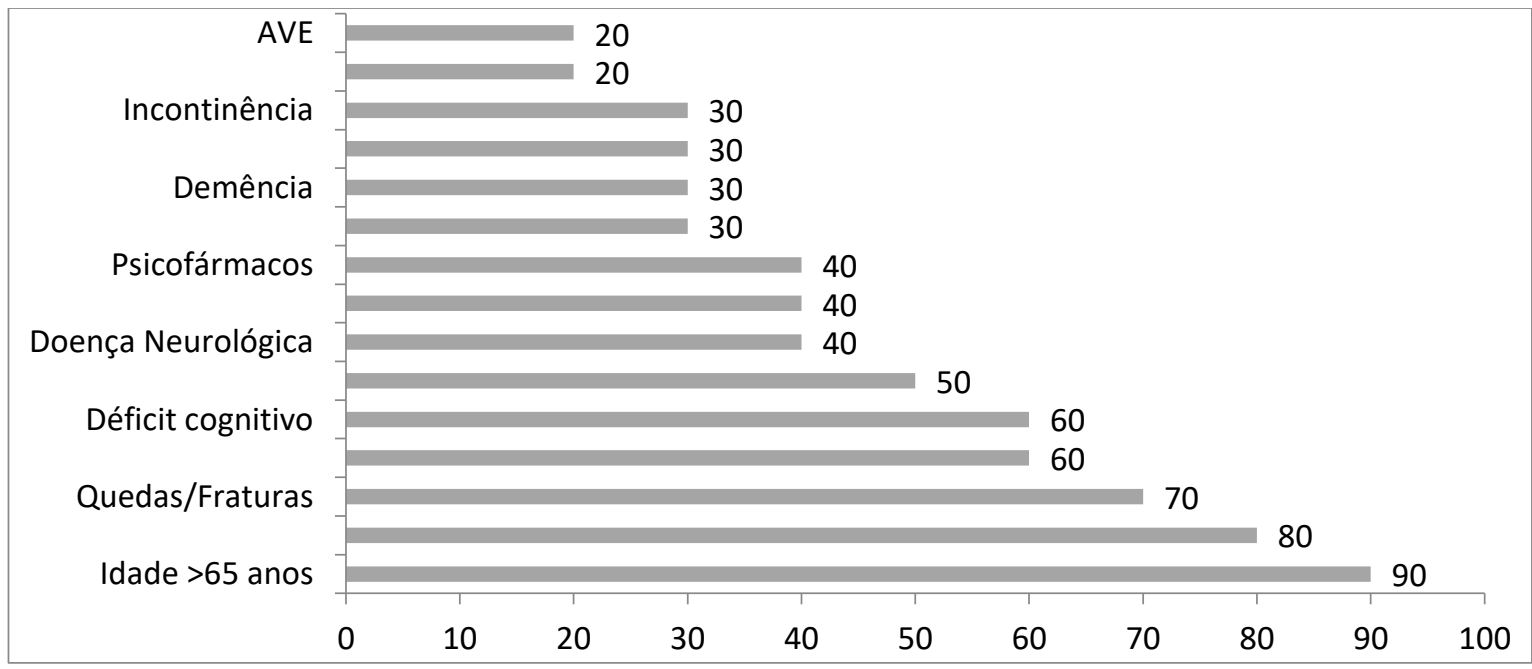

Fonte: Protocolo de pesquisa 
Com relação aos Fatores Precipitantes, o de maior prevalência foi o uso de Inibidores de bombas de Prótons - IBP com prevalência de 100\%, em seguida ficou Infecção e Dor, ambas com prevalência de $80 \%$ e logo depois Delírium Agudo, Estresse emocional e Anemia com 60\% de prevalência (Gráfico 3).

Gráfico 3 - Distribuição dos Fatores Precipitantes apresentados pelos pacientes com Delírium na Clínica médica da FSCMPA entre junho-2017 a junho-2018.



Fonte: Protocolo de pesquisa

\section{DISCUSSÃO}

A incidência de Delírium encontrada nas enfermarias de clínica médica foi de $28 \%$, taxa esta que está dentro da média encontrada em outros trabalhos como no de Souza, o qual mostra que a taxa de incidência de delírium na hospitalização varia de 6 a 56\% (PEREIRA e LOPES 2018).

Este estudo identificou que dentre os pacientes que apresentaram delírium, $50 \%$ eram do gênero feminino e $50 \%$ do gênero masculino, fato que diverge dos resultados encontrados por Pereira et al (2016), onde a maior prevalência foi em indivíduos do gênero masculino.

Pereira e Cols (2016) também demonstrou a maioria dos indivíduos que apresentaram delírium possuíam idade próxima a 67 anos, o mesmo foi visto neste trabalho, no qual $90 \%$ dos indivíduos possuíam idade superior a 65 anos.

Neste estudo o delírium mais prevalente foi do tipo hipoativo (60\%), seguido do hiperativo (30\%) e por fim do misto $(10 \%)$. Os mesmos resultados foram vistos por Vitodo e cols mostrando a prevalência do delírium hipoativo em $50 \%$ dos pacientes. 
Todos os pacientes nesse estudo possuíam tanto fatores precipitantes como fatores predisponentes, o que corrobora para os achados de Souza, 2015, onde os fatores precipitantes e predisponentes podem levar ao quadro de delírium de modo independente e cumulativo (MORI et al., 2016)

Os fatores predisponentes mais comumente descritos pelos trabalhos mostram que a idade acima de 65 anos, múltiplas comorbidades e déficit cognitivo. O que é compatível com os achados deste trabalho, sendo a idade avançada presente em $90 \%$ dos pacientes, as múltiplas comorbidades em $50 \%$ e os déficits cognitivos em $60 \%$ dos pacientes avaliados (SAXENA, 2009; INOUYE 2012; YOUG, 2011; MITTAL, 2007; BUROCK, 2013).

Considerando a ordem decrescente dos principais fatores predisponentes encontrados nesta pesquisa - idade $>65$ anos, polifarmácia, déficit visual - O estudo de Pereira e Lopes (2018) apontou especificamente o uso de morfina como estando relacionado ao delirium. Cerveira et al (2017) também informaram que a polifarmácia é o fator de risco para delirium mais comumente independente e reversível. Rowland e Pedley (2011) comentam que o déficit visual põe mais o paciente em risco ao delirium, o que favorece o achado desta pesquisa.

Os fatores Precipitantes mais comuns nos idosos incluem fármacos, infecções e alterações laboratoriais, seguidos de eventos cardiovasculares traumatismos e iatrogenia, sobretudo cirurgia. O que foi compatível com os achados deste estudo, no qual $100 \%$ dos pacientes utilizavam fármaco da classe IBP e $60 \%$ utilizavam AntiHipertensivos, $80 \%$ possuíam algum tipo de infecção e dor, e 50\% possuíam alterações laboratoriais (alterações principalmente de glicemia e eletrolítica) (SAXENAM 2009, HAN 2010, HAN 2011, HALL, 2012).

Analisando-se os principais fatores precipitantes achados na população estudada - variações da glicemia, infecções, dor, anemia e hipoxemia - O fator "infecção" coincide com os achados de Pereira e Lopes (2018) e com o comentário inicial de Cerveira et al (2017). Variações da glicemia, anemia e hipoxemia foram apontados como fatores de risco, entre outros, por Freitas et al (2013).

O tratamento do delirium envolve não somente controle farmacológico, mas sua base esta pautada na identificação e controle dos fatores precipitantes e predisponentes a fim de que o mecanismo desencadeante do processo confusional agudo cesse e que o paciente se restabeleça (MORAES 2008).

O estudo demostra como o desenvolvimento de Delírium é multifatorial e muito prevalente nos idosos hospitalizados. O conhecimento desses fatores de risco e identificação precoce dos mesmos é capaz de reduzir o risco de ocorrência de Delirium, bem como de suas complicações como quedas, piora clínica e maior tempo de hospitalização, levando assim a uma melhor assistência ao paciente e intervindo precocemente nas complicações.

\section{CONCLUSÃo}

A maioria dos pacientes analisados possuía idade acima de 65 anos. O sexo masculino foi o mais prevalente. A maioria dos pacientes apresentou Delirium do tipo hipoativo e todos os pacientes apresentavam Fatores Precipitantes e Predisponentes. É necessário aperfeiçoar o exame clínico da equipe de saúde que lida diretamente com os pacientes idosos, a fim de detectar precocemente os sinais que sugerem uma evolução para o estado de delirium e estabelecer medidas terapêuticas e preventivas, para melhorar 0 atendimento aos pacientes e evitar as complicações. 


\section{REFERÊNCIAS}

1. BARBOSA FT, CUNHA RM, PINTO ALCLTI. Delirium pós-operatório em idosos. Rev. Bras. Anestesiologia, 2008; 58(6)

2. BRUNNER \& SUDDARTH. Tratado de enfermagem médico-cirúrgica. Tradução PAULO AFD et al. $12^{\underline{a}}$ ed. Rio de Janeiro: Guanabara Koogan, 2011.

3. BUROCK JM. Delirium in the elderly. Medicine \& Health/Rhode Island, 2012, 95(7): 214-219.

4. CÁSSIO MC, BOTTTINO JL, BLAY SL. Demência e transtorno cognitivo em idosos. Rio de Janeiro: Editora Guanabara Koogan, 2006.

5. CHAGAS NMS, BORGES DGS, CHAGAS MHN. Delirium como fator de risco para demência em idosos: uma atualização. J Bras Psiquiatria, 2016, 65(1): 94-8.

6. DI TOMMASO ABG, MORAES NS, CRUZ EC, et al. Guia prático de geriatria. $1^{\text {a }}$ ed. Rio de Janeiro: Editora Guanabara Koogan, 2016.

7. FREITAS EV, PY L, GORZONI ML, et al. Tratado de geriatria e gerontologia. $4^{\mathrm{a}}$ ed. Rio de Janeiro: Editora Guanabara Koogan, 2016.

8. FREITAS EV, PY L, GORZONI ML, et al. Manual prático de geriatria. 1a ed. Rio de Janeiro: A. C. Farmacêuta, 2012.

9. GORZONI ML, FABBRI RMA. Livro de bolso de Geriatria. São Paulo: Editora Atheneu, 2013.

10. HALL RJ. Delirium detection and monitoring outside the ICU. Best Practice \& Research Clinical Anaesthesiology, 2012367-383.

11. HAN JH, WILSON A, ELY EW. Delirium in the older emergency department patient - a quiet epidemic. Emerg Med Clin North Am, 2010; 28(3): 611-631

12. HAN JH. Delirium in older emergency department patients is an independent predictor of hospital length of stay. Society for Academic Emergency Medicine, 2011; 18(5): 451-457.

13. INOUYE SK, WESTENDORP RGJ, SACZYNSKI JS. Delirium in elderly people. The Lancet, 2013; 383(9920): 911-922.

14. LÔBO RR, SILVA FILHO SRB, LIMA NKC, et al.Delirium. Medicina (Ribeirão Preto) 2010;43(3): 249-57. Simpósio: Condutas em enfermaria de clinica médica de hospital de média complexidade - Parte 2, capítulo IV.

15. MITTAL V. Delirium in the Elderly: a comprehensive review. American Journal of Alzheimer's Disease \& Other Dementias, 2011; 26(2): 97-109.

16. MORAES, EN. Princípios básicos de geriatria e gerontologia. Belo Horizonte: Coopmed, 2008;

17. MORI S, TAKEDA JRT, CARRARA FSA, et al. Incidência e fatores relacionados ao delirium em unidade de terapia intensiva. Rev Esc Enferm USP, 2016; 50(4): 587-593

18. NETO JT, PINTARELLI VL, YAMATTO TH. A beira do leito: geriatria e gerontologia na prática hospitalar. São Paulo: Manoele, 2007.

19. PARSONS PE, WIENER- KRONISH JP. Segredos em terapia intensiva: respostas necessárias ao dia-a-dia em rounds, na clínica, em exames orais e escritos. Trad. Eliézer Silva e Alejandradel Pilar Gallardo Garrido. 2ª ed. Porto Alegre: Artmed, 2003;

20. PESSOA RF, NÁCUL FE. Delirium em pacientes críticos. Revista Brasileira Terapia Intensiva, 2006; 18(2).

21. PITROWSKY MT, SHINOTSUKA CR, SOARES M, et al. Importância da monitorização do delirium na unidade de terapia intensiva. Revista Brasileira Terapia Intensiva, 2010; 22(3): 274-279.

22. SANTOS, F.S. Mecanismos fisiopatológicos do delirium. Revista Psiquiatria Clínica, 2005; 32(3): 104-112.

23. SAXENA S, LAWLEY D. Delirium in the elderly: a clinical review. Postgraduate Medical Journal, 2009; 85: 405-413.

24. SCHLINDWEIN-ZANINI R. Demência no idoso: aspectos neuropsicológicos. Revista Neurocienc, 2010; 18(2): 220226.

25. SILVA, RC. Incidência e fatores de risco associados a delirium em pacientes no pós-operatório de cirurgias abdominais internados no Hospital Universitário de Florianópolis. Monografia (Trabalho de Conclusão de Curso) - Curso de Graduação em Medicina. Universidade Federal de Santa Catarina, Florianópolis, 2004; 38p.

26. SOUSA-MUÑOZ RL, TAGUSHI LC, MARTINS GCCS, et al. Prevalência e fatores associados com ocorrência de delirium em adultos e idosos internados. Revista Brasileira Clínica Medica, 2012; 10(4): 285-90.

27. WACKER P, NUNES PV, FORLENZA OV. Delirium: uma perspectiva histórica. Revista Psiquiatria Clínica, 2005; 32(3): 97-103.

28. YOUG J, INOUYE SK, Delirium in older people. BMJ, 2007; 842-846. 